\title{
An Optimal Control Problem Governed by a Kirchhoff-Type Variational Inequality
}

\author{
Chengfu Wang, Pengcheng $W u(\mathbb{D}$, and Yuying Zhou $\mathbb{i}$ \\ Department of Mathematics, Soochow University, Suzhou 215006, China \\ Correspondence should be addressed to Yuying Zhou; yuyingz@suda.edu.cn
}

Received 15 September 2019; Accepted 13 January 2020; Published 18 February 2020

Academic Editor: Sheng Zhang

Copyright ( $\odot 2020$ Chengfu Wang et al. This is an open access article distributed under the Creative Commons Attribution License, which permits unrestricted use, distribution, and reproduction in any medium, provided the original work is properly cited.

This paper is concerned with an optimal control problem governed by a Kirchhoff-type variational inequality. The existence of multiplicity solutions for the Kirchhoff-type variational inequality is established by using some nonlinear analysis techniques and the variational method, and the existence results of an optimal control for the optimal control problem governed by a Kirchhofftype variational inequality are derived.

\section{Introduction}

Let $\Omega$ be a bounded domain of $\mathbb{R}^{N}$ with a smooth boundary $\partial \Omega, \mathcal{U}$ be a nonempty bounded closed and convex subset of the space $L^{q}(\Omega)\left(1<q<\min \left\{4,2^{*}\right\}, 2^{*}=(2 N / N-2)\right)$, $\psi: \Omega \longrightarrow[0,+\infty)$ be a proper and convex function, and

$$
\mathscr{K}_{\psi}=\left\{u \in H_{0}^{1}(\Omega): u(x) \leq \psi(x) \text {, a.e. } x \in \Omega\right\} .
$$

Let $H_{0}^{1}(\Omega)$ be endowed with the norm $\|\cdot\|$, and let $g: \Omega \times$ $\mathbb{R} \longrightarrow \mathbb{R}$ and $l: \Omega \times \mathbb{R} \longrightarrow \mathbb{R}$. The objective functional $\mathbf{J}: \mathscr{U} \times \mathscr{K}_{\psi} \longrightarrow \mathbb{R}$ is defined by

$$
\mathbf{J}(w, u)=\int_{\Omega} g(x, u) d x+\int_{\Omega} l(x, w) \mathrm{d} x, \quad \forall(w, u) \in \mathscr{U} \times \mathscr{K}_{\psi} .
$$

In this paper, we will be discussing the following optimal control problem governed by a state variational inequality:

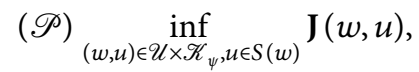

where $S(w)$ is the solution set of the following Kirchhofftype variational inequality: for each $w \in \mathcal{U}$, find $u=u(w) \in \mathscr{K}_{\psi}$ (the state function of the system), such that

$$
h(\|u\|) \int_{\Omega} \nabla u(\nabla v-\nabla u) \mathrm{d} x \geq \lambda \int_{\Omega} f(x, u)(v-u) \mathrm{d} x-\mu \int_{\Omega} \tau(w)(v-u) \mathrm{d} x, \quad \forall v \in \mathscr{K}_{\psi},
$$

where $\lambda>0, \quad \mu>0, \quad f(x, t): \Omega \times \mathbb{R} \longrightarrow \mathbb{R}, \quad \tau: \mathcal{U} \longrightarrow$ $L^{q^{\prime}}(\Omega)\left(q^{\prime}=(q / q-1)\right)$ and $h: \mathbb{R} \longrightarrow \mathbb{R}$ satisfies, $\left(h_{1}\right) h$ is continuous on $\mathbb{R} ;\left(h_{2}\right)$ there exists $a>0$ such that $h(t)>a$ for all $t \in \mathbb{R} ;\left(h_{3}\right)$ there exists $b>0$ such that $\lim _{t \rightarrow+\infty} h(t) / t^{2}=b$.

A typical example of $h$ is $h(t)=a+b t^{2}$. Then, $h$ satisfies $\left(h_{1}\right)-\left(h_{3}\right)$,

$$
\|h(u)\|=a+b \int_{\Omega}|\nabla u|^{2},
$$

and the variational inequality (4) will become to be the usual variational inequality of the Kirchhoff type: for each $w \in \mathcal{U}$, find $u=u(w) \in \mathscr{K}_{\psi}$, such that 


$$
\left(a+b \int_{\Omega}|\nabla u|^{2} \mathrm{~d} x\right) \int_{\Omega} \nabla u(\nabla v-\nabla u) \mathrm{d} x \geq \lambda \int_{\Omega} f(x, u)(v-u) \mathrm{d} x-\mu \int_{\Omega} \tau(w)(v-u) \mathrm{d} x, \quad \forall v \in \mathscr{K}_{\psi} .
$$

The Kirchhoff Dirichlet problem was first proposed by Kirchhoff by taking into account a differential equation describing the changes in length of the string produced by transverse vibrations for free vibrations of elastic strings. For more details on the physical and mathematical background of Kirchhoff-type problems, we refer the readers to the papers $[1-3]$ and the references therein. By variational methods, many interesting results about the existence multiplicity of solutions for Kirchhoff-type problems have been established in the last ten years, see, e.g., [1-7] and the references therein.

The study of variational inequalities like (4) with $b=0$ and related optimal control problems was proposed by Lions [8-10], and this topic has been widely studied by many authors in different aspects (cf. [11-23]). One of the most important methods is the approximation of the variational inequality by an equation where the maximal monotone operator (in this case, the subdifferential of a Lipschitz function) is approached by a differentiable singlevalue mapping with Moreau-Yosida approximation techniques. This method, mainly due to Barbu [11], leads to several existence results and to first-order optimality systems. Lou [12] discussed the regularity of an obstacle control problem, wherein the variational inequality is associated to the Laplace operator. Lou [13] considered the existence and regularity of the control problem governed by the quasilinear elliptic variational inequality. Bergounioux and Lenhart [15] studied obstacle optimal control for semilinear and bilateral obstacle problems. Chen et al. [21] studied an optimal control problem for quasilinear elliptic variational inequality. Ye and Chen [16] studied the existence and necessary condition of an optimal control problem for a quasilinear elliptic obstacle variational inequality in which the obstacle was taken as the control and the cost functional were specific. Zhou et al. [17] established the existence of the optimal control for an optimal control problem governed by an abstract variational inequality and obtained the existence of the optimal control for the optimal control problem governed by a quasilinear elliptic variational inequality with an obstacle. By using nonlinear Lagrangian methods, Zhou et al. [18] studied an optimal control problem where the state of the system is defined by a variational inequality problem for monotonetype mappings. Khan and Sama [19] obtained the existence of an optimal control for a quasivariational inequality with multivalued pseudomonotone maps. Chen et al. [20] studied an optimal control problem for a quasilinear elliptic variational inequality with source term, established the existence results, and derived the optimality system for this optimal control problem. In [22], Migorski et al. investigated an inverse problem of identifying the material parameter in an implicit obstacle problem given by an operator of $p$-Laplacian type. In [23], Khan et al. studied inverse problems of identifying a variable parameter in variational and quasivariational inequalities.
The purpose of the present paper is to investigate the optimal control problem governed by the state Kirchhofftype variational inequality (4), i.e., the problem $(\mathscr{P})$ in the case of $b>0$. This case is more complicated since the socalled nonlocal term $b \int_{\Omega}|\nabla u|^{2} d x \Delta u$ is involving in the variational inequality. To the best of our knowledge, the study on optimal control problems controlled by the state Kirchhoff-type variational inequality is still lacking in mathematics literatures. Our first intention is to establish the existence and multiplicity of solutions for the inequality (4) by using some nonlinear analysis techniques and the variational method. Then, as an application, we obtain the existence of solutions for the optimal control problem $(\mathscr{P})$.

The paper is structured as follows. Section 2 contains some basic definitions and preliminary facts needed in the sequel. In Section 3, we shall show that there exist at least two solutions of the Kirchhoff-type variational inequality (4) when some suitable conditions on $f, h$, and $\tau$ are satisfied. In Section 4 , we apply the obtained results to study the optimal control problem governed by the state variational inequality (4) and obtain the existence of solutions for the problem $(\mathscr{P})$.

\section{Preliminary}

Let $X$ be a Banach space and $X^{*}$ its dual. The following definitions and theorems can be found in, e.g., $[24,25]$.

Definition 1. Let $X$ be a Banach space, $\varphi: X \longrightarrow \mathbb{R}$ be a continuously differentiable functional, and let $\psi: X \longrightarrow \mathbb{R} \cup\{+\infty\}$ be a proper (i.e., $\neq+\infty$ ), convex, and lower semicontinuous functional. The functional $\varphi+\psi: X \longrightarrow \mathbb{R} \cup\{+\infty\}$ is called Szulkin-type functional. $\varphi+\psi$ is said to satisfy the Palais-Smale condition (the (PS). condition for short), if every sequence $\left\{u_{n}\right\} \subset X$ with $\varphi\left(u_{n}\right)+\psi\left(u_{n}\right)$ bounded and for which there exists a sequence $\left\{\varepsilon_{n}\right\} \subset \mathbb{R}_{+}, \varepsilon_{n} \backslash 0$, such that

$$
\left\langle\varphi^{\prime}\left(u_{n}\right), v-u_{n}\right\rangle+\psi(v)-\psi\left(u_{n}\right) \geq-\varepsilon_{n}\left\|v-u_{n}\right\|, \quad \forall v \in X,
$$

contains a (strongly) convergent subsequence in $X$.

Definition 2. Let $I=\varphi+\psi: X \longrightarrow X \cup\{+\infty\}$ be a Szulkintype functional. A point $u \in X$ is said to be a critical point of $I=\varphi+\psi$ if

$$
\left\langle\varphi^{\prime}(u), v-u\right\rangle+\psi(v)-\psi(u) \geq 0, \quad \forall v \in X .
$$

The value $c$ of I at a critical point $u \in X$ is said to be a critical value of $I$, that is, $I(u)=c$.

Theorem 1. Let $I=\varphi+\psi: X \longrightarrow X \cup\{+\infty\}$ be a Szulkintype functional which is bounded below. If I satisfies the (PS) condition for $c=\inf _{u \in X} I(u)$, then $c$ is a critical value. 
Theorem 2. Let $I=\varphi+\psi: X \longrightarrow X \cup\{+\infty\}$ be a Szulkintype functional and assume that

(1) $I(u) \geq d_{0}$ for all $\|u\|=\rho$ for some $d_{0}, \rho>0$, and $I(0)=0$

(2) There is $e \in X$ with $\|e\|>\rho$ and $I(e) \leq 0$

If I satisfies the (PS) condition, then I has a critical point $u^{*} \in X$ with critical value $c_{0}=I\left(u^{*}\right)$ such that

$$
\begin{aligned}
& c_{0} \geq d_{0}, \\
& c_{0}=\inf _{g \in \Gamma} \sup _{t \in[0,1]} I(\gamma(t)),
\end{aligned}
$$

where

$$
\Gamma=\{g \in C([0,1], X): g(0)=0, g(1)=e\} .
$$

Let $f(x, t): \Omega \times \mathbb{R} \longrightarrow \mathbb{R}$ be a function. We denote by

$$
F(x, t)=\int_{0}^{t} f(x, s) \mathrm{d} s .
$$

The hypotheses on the $f$ are the following:

$\left(f_{1}\right) f(x, t)$ is measurable in $x$ for every $t \in \mathbb{R}$ and continuous in $t$ for a.e. $x \in \Omega$;

$\left(f_{2}\right)|f(x, t)| \leq \alpha(x)+\beta(x)|t|^{q-1}$ uniformly for almost all $x \in \Omega$, where

$$
\begin{aligned}
& \alpha \in L^{\infty}(\Omega), \\
& \beta \in L^{\infty}(\Omega), \\
& 1<q<\min \left\{4,2^{*}\right\}, \\
& 2^{*}=\frac{2 N}{N-2} .
\end{aligned}
$$

$\left(f_{3}\right)$ There exists a constant $\gamma>\max \{2, q\}$ and a function $\chi \in L^{\infty}(\Omega)$ such that

$$
\limsup _{t \longrightarrow 0} \frac{F(x, t)}{|t|^{\gamma}} \leq \chi(x)
$$

uniformly for almost all $x \in \Omega$;

$\left(f_{4}\right)$ There exist $x_{0} \in \Omega, u_{0} \in \mathbb{R}_{+}$and $\delta>0$ such that

$$
F\left(x, u_{0}\right)>\eta_{0}, \quad \text { a.e. } x \in U\left(x_{0}, \delta\right),
$$

where $\eta_{0}$ is a positive constant and $U\left(x_{0}, \delta\right)=$ $\left\{x \in \Omega:\left|x-x_{0}\right|<\delta\right\} \subset \Omega$.

Let $h: \mathbb{R} \longrightarrow \mathbb{R}^{+}$satisfy the conditions $\left(h_{1}\right)-\left(h_{3}\right)$. We denote by

$$
H(t)=\int_{0}^{t} h(s) \mathrm{d} s, \quad \widehat{H}(t)=\int_{0}^{t} H(s) \mathrm{d} s .
$$

Let $\lambda>0, \mu>0$, and let us introduce the Euler functional $\varphi_{\lambda, \mu}: H_{0}^{1}(\Omega) \longrightarrow \mathbb{R}$ corresponding to the Kirchhoff-type variational inequality problem (4) as

$$
\begin{aligned}
\varphi_{\lambda, \mu}(u)= & \|u\| H(\|u\|)-\widehat{H}(\|u\|)-\lambda \int_{\Omega} F(x, u) \mathrm{d} x \\
& +\mu \int_{\Omega} \tau(\omega) u \mathrm{~d} x .
\end{aligned}
$$

Let $\mathscr{K}_{\psi}$ be defined by (1). We define the indicator functional of the set $\mathscr{K}_{\psi}$ by

$$
\phi(u)= \begin{cases}0, & \text { if } u \in \mathscr{K}_{\psi}, \\ +\infty, & \text { if } u \notin \mathscr{K}_{\psi} .\end{cases}
$$

Denote

$$
I_{\lambda, \mu}=\varphi_{\lambda, \mu}+\phi
$$

Obviously, $I_{\lambda, \mu}$ is a Szulkin-type functional.

Remark 1 . For any $s \in \mathbb{R}^{+}$, if $h(s)=a+b s^{2}$, then $h$ satisfies $\left(h_{1}\right)-\left(h_{3}\right)$, and

$$
t H(t)-\widehat{H}(t)=\frac{a}{2} t^{2}+\frac{b}{4} t^{4}
$$

The variational inequality (4) will become to be the classic Kirchhoff-type variational inequality: find $u=$ $u(w) \in \mathscr{K}_{\psi}$, such that

$$
\left(a+b \int_{\Omega}|\nabla u|^{2} \mathrm{~d} x\right) \int_{\Omega} \nabla u(\nabla v-\nabla u) \mathrm{d} x \geq \lambda \int_{\Omega} f(x, u)(v-u) \mathrm{d} x-\mu \int_{\Omega} \tau(w)(v-u) \mathrm{d} x, \quad \forall v \in K_{\psi}
$$

\section{Existence of Multiple Solutions for a Kirchhoff-Type Variational Inequality}

As usual, we denote “ $\longrightarrow$ ” and “ $\rightarrow$ " by the strong and weak convergence in the space $H_{0}^{1}(\Omega)$.

Proposition 1. Let $\mathcal{U}$ be a nonempty bounded closed and convex subset of the space $L^{q}(\Omega)\left(1<q<\min \left\{4,2^{*}\right\}\right)$. If $f: \Omega \times \mathbb{R} \longrightarrow \mathbb{R}$ satisfies $\left(f_{1}\right),\left(f_{2}\right), h: \mathbb{R} \longrightarrow \mathbb{R}$ satisfies conditions $\left(h_{1}\right)-\left(h_{3}\right)$, and $\tau: \mathcal{U} \longrightarrow L^{q^{\prime}}(\Omega)\left(q^{\prime}=q / q-1\right)$ is a weakly continuous mapping. Then, every critical point $u \in H_{0}^{1}(\Omega)$ of $I_{\lambda, \mu}=\varphi_{\lambda, \mu}+\phi$ is a solution of $(4)$, where $\varphi_{\lambda, \mu}$ and $I_{\lambda, \mu}$ are defined by (16) and (18), respectively.
Proof. It is easy to see that $\varphi_{\lambda, \mu} \in C^{1}\left(H_{0}^{1}(\Omega), \mathbb{R}\right)$ and for each $u, v \in H_{0}^{1}(\Omega)$,

$$
\begin{aligned}
\left\langle\varphi_{\lambda, \mu}^{\prime}(u), v\right\rangle= & h(\|u\|) \int_{\Omega} \nabla u \nabla v \mathrm{~d} x-\lambda \int_{\Omega} f(x, u) v \mathrm{~d} x \\
& +\mu \int_{\Omega} \tau(\omega) v \mathrm{~d} x .
\end{aligned}
$$

If $u \in H_{0}^{1}(\Omega)$ is a critical point of $I_{\lambda, \mu}$, from Definition 2, we obtain

$$
\left\langle\varphi_{\lambda, \mu}^{\prime}(u), v-u\right\rangle+\phi(v)-\phi(u) \geq 0, \quad \forall v \in H_{0}^{1}(\Omega) .
$$
holds.

It follows from (17), (21), and (22) that $u \in \mathscr{K}_{\psi}$ and (4) 
Proposition 2. Let $\mathcal{U}$ be a nonempty bounded, closed, and convex subset of the space $L^{q}(\Omega)\left(1<q<\min \left\{4,2^{*}\right\}\right)$. If $f: \Omega \times \mathbb{R} \longrightarrow \mathbb{R}$ satisfies $\left(f_{1}\right),\left(f_{2}\right), h: \mathbb{R} \longrightarrow \mathbb{R}$ satisfies conditions $\left(h_{1}\right)-\left(h_{3}\right)$, and $\tau: \mathcal{U} \longrightarrow L^{q^{\prime}}(\Omega)\left(q^{\prime}=q / q-1\right)$ is a weakly continuous mapping such that $\tau(\mathcal{U})$ is a bounded set. Then, $I_{\lambda, \mu}$ is coercive in the sense of $I_{\lambda, \mu}(u) \longrightarrow+\infty$ as $\|u\| \longrightarrow+\infty$ and bounded from below in $\mathscr{K}_{\psi}$, where $\mathscr{K}_{\psi}$ is defined by (1) and $I_{\lambda, \mu}$ is defined (18).

Proof. By $\left(f_{2}\right)$, Hölder inequality, we have

$$
\begin{aligned}
\left|\int_{\Omega} F(x, u(x)) \mathrm{d} x\right| & \leq \int_{\Omega} \alpha(x)|u(x)| \mathrm{d} x+\int_{\Omega} \frac{\beta(x)}{q}|u(x)|^{q} \mathrm{~d} x \\
& \leq|\Omega|^{1 / q^{\prime}}\|\alpha\|_{L^{\infty}(\Omega)}\|u\|_{L^{q}(\Omega)}+\frac{\|\beta\|_{L^{\infty}(\Omega)}}{q}\|u\|_{L^{q}(\Omega)}^{q} \\
& \leq c_{1}\|u\|+c_{2}\|u\|^{q}
\end{aligned}
$$

where $c_{1}$ and $c_{2}$ are positive constants.

Since $\tau(U)$ is bounded, there exists $\rho>0$, such that

$$
\|\tau(w)\|_{L^{q^{\prime}}}<\rho, \quad \forall w \in U
$$

Thus,

$$
\begin{aligned}
\left|\int_{\Omega} \tau(w) u \mathrm{~d} x\right| & \leq\left(\int_{\Omega}|\tau(w)|^{q^{\prime}}\right)^{1 / q^{\prime}}\left(\int_{\Omega}|u|^{q} \mathrm{~d} x\right)^{1 / q} \\
& \leq \rho\|u\|_{L^{q}(\Omega)} \leq c_{3}\|u\|
\end{aligned}
$$

where $c_{3}$ is a positive constant.

By $\left(h_{3}\right)$, we have

$$
\lim _{t \rightarrow+\infty} \frac{t H(t)-\hat{H}(t)}{t^{4}}=\lim _{t \rightarrow+\infty} \frac{h(t)}{4 t^{2}}=\frac{b}{4} .
$$

Let $u \in \mathscr{K}_{\psi}$. By (16), (18), (23), and (25), we have

$$
\begin{gathered}
I_{\lambda, \mu}(u)=\varphi_{\lambda, \mu}(u) \geq\|u\| H(\|u\|)-\widehat{H}(\|u\|)-\left(\lambda c_{1}+\mu c_{3}\right)\|u\| \\
-c_{2} \lambda\|u\|^{q} .
\end{gathered}
$$

By noting that $1<q<4$ and from (26), we obtain that $I_{\lambda, \mu}$ is coercive and $I_{\lambda, \mu}$ is bounded from below in $\mathscr{K}_{\psi}$. The proof is complete.

Proposition 3. Let all conditions in Proposition 2 be satisfied. Then, $I_{\lambda, \mu}$ satisfies the (PS) condition in the sense of Definition 1.

Proof. Let $\left\{u_{n}\right\} \subset H_{0}^{1}(\Omega)$ such that $\left\{I_{\lambda, \mu}\left(u_{n}\right)\right\}$ is bounded, and there exists $\left\{\varepsilon_{n}\right\} \subset \mathbb{R}_{+}$with $\varepsilon_{n} \searrow 0$ and

$$
\left\langle\varphi_{\lambda, \mu}^{\prime}\left(u_{n}\right), v-u_{n}\right\rangle+\phi(v)-\phi\left(u_{n}\right) \geq-\varepsilon_{n}\left\|v-u_{n}\right\|, \quad \forall v \in H_{0}^{1}(\Omega) .
$$

From (17), it can be noted that it is easy to obtain $\left\{u_{n}\right\} \subset \mathscr{K}_{\psi}$. By Proposition $2, I_{\lambda, \mu}$ is coercive, and then $\left\{u_{n}\right\}$ is bounded in $\mathscr{K}_{\psi} \subset H_{0}^{1}(\Omega)$. Hence, by the Sobolev embedding theorem, we may assume that going to a subsequence,

$$
\begin{array}{ll}
u_{n} \longrightarrow u, & \text { in } H_{0}^{1}(\Omega) ; \\
u_{n} \longrightarrow u, & \text { in } L^{q}(\Omega),
\end{array}
$$

since $1<q<\min \left\{4,2^{*}\right\}$.

As $\mathscr{K}_{\psi}$ is weakly closed, $u \in \mathscr{K}_{\psi}$. Setting $v=u$ in (28) and combining with (21), we have

$$
\begin{aligned}
& h\left(\left\|u_{n}\right\|\right) \int_{\Omega}\left[\nabla u_{n}\left(\nabla u_{n}-\nabla u\right)\right] \mathrm{d} x-\lambda \int_{\Omega} f\left(x, u_{n}\right)\left(u_{n}-u\right) \mathrm{d} x \\
& \quad \leq \varepsilon_{n}\left\|u_{n}-u\right\|-\mu \int_{\Omega} \tau(w)\left(u_{n}-u\right) \mathrm{d} x .
\end{aligned}
$$

Thus,

$$
\begin{aligned}
& h\left(\left\|u_{n}\right\|\right)\left\|u-u_{n}\right\|^{2} \\
& =h\left(\left\|u_{n}\right\|\right) \int_{\Omega}\left[\nabla u\left(\nabla u-\nabla u_{n}\right)+\nabla u_{n}\left(\nabla u_{n}-\nabla u\right)\right] \mathrm{d} x \\
& \leq h\left(\left\|u_{n}\right\|\right) \int_{\Omega} \nabla u\left(\nabla u-\nabla u_{n}\right) \mathrm{d} x+\lambda \int_{\Omega} f\left(x, u_{n}\right)\left(u_{n}-u\right) \mathrm{d} x \\
& \quad+\varepsilon_{n}\left\|u-u_{n}\right\|-\mu \int_{\Omega} \tau(w)\left(u-u_{n}\right) \mathrm{d} x .
\end{aligned}
$$

By the Hölder inequality and the condition $\left(f_{2}\right)$, for $\left\{u_{n}\right\} \subset \mathscr{K}_{\psi}$,

$$
\begin{aligned}
& \int_{\Omega} f\left(x, u_{n}\right)\left(u_{n}-u\right) \\
& \quad \leq \int_{\Omega}\left(\alpha(x)+\beta(x)\left|u_{n}\right|^{q-1}\right)\left|u_{n}-u\right| \mathrm{d} x \\
& \quad \leq\|\alpha\|_{L^{\infty}}\left\|u_{n}-u\right\|_{L^{1}}+\|\beta\|_{L^{\infty}}\left\|u_{n}\right\|_{L^{q-1}}\left\|u_{n}-u\right\|_{L^{q}} .
\end{aligned}
$$

Note that $\varepsilon_{n} \searrow 0($ as $n \longrightarrow \infty),\left\{u_{n}\right\}$ is bounded in $H_{0}^{1}(\Omega)$ and $h$ satisfies $\left(h_{1}\right)-\left(h_{2}\right)$; by $(29)-(32)$, we have

$$
0 \leq \lim _{n \longrightarrow \infty} a\left\|u-u_{n}\right\|^{2} \leq \lim _{n \longrightarrow \infty} h\left(\left\|u_{n}\right\|\right)\left\|u-u_{n}\right\|^{2} \leq 0 .
$$

Therefore $u_{n} \longrightarrow u$ in $H_{0}^{1}(\Omega)$.

Theorem 3. Let $U$ be a nonempty bounded closed and convex subset of the space $L^{q}(\Omega)\left(1<q<\min \left\{4,2^{*}\right\}\right)$. If $f: \Omega \times \mathbb{R} \longrightarrow \mathbb{R}$ satisfies $\left(f_{1}\right)-\left(f_{4}\right), h: \mathbb{R} \longrightarrow \mathbb{R}$ satisfies the conditions $\left(h_{1}\right)-\left(h_{3}\right)$, and $\tau: \mathscr{U} \longrightarrow L^{q^{\prime}}(\Omega)$ is a weakly continuous mapping such that $\tau(\mathcal{U})$ is a bounded set. Then, there exists $\lambda_{*}>0,0<\mu_{*}<1$, such that for each $\lambda>\lambda_{*}, \mu<\mu_{*}$, the Kirchhoff-type variational inequality (4) has two solutions $u_{\lambda}, v_{\lambda}$, satisfying $I_{\lambda}\left(u_{\lambda}\right)<0<I_{\lambda}\left(v_{\lambda}\right)$.

Proof. Noting the conditions $\left(f_{3}\right)$ and $\left(f_{4}\right)$, we have

$$
0 \leq M_{1}=\|\alpha\|_{L^{\infty}}\left|u_{0}\right|+\frac{1}{q}\|\beta\|_{L^{\infty}}\left|u_{0}\right|^{q}<+\infty .
$$

Denote by

$$
t_{0}=\frac{1}{2}\left(\left(\frac{M_{1}}{\eta_{0}+M_{1}}\right)^{1 / N}+1\right),
$$

where $\eta_{0}$ is given in $\left(f_{4}\right)$. Let us define 


$$
u_{t_{0}}(x)= \begin{cases}0, & x \in \Omega U\left(x_{0}, \delta\right), \\ u_{0}, & x \in U\left(x_{0}, t_{0} \delta\right), \\ \frac{u_{0}}{\delta\left(1-t_{0}\right)}\left(\delta-\left|x-x_{0}\right|\right), & x \in U\left(x_{0}, \delta\right) \backslash U\left(x_{0}, t_{0} \delta\right),\end{cases}
$$

where $x_{0}$ is given in $\left(f_{4}\right)$. Then,

$$
\begin{aligned}
\left\|u_{t_{0}}\right\|^{2} & =\int_{\Omega}\left|\nabla u_{t_{0}}\right|^{2} \mathrm{~d} x=\int_{B\left(x_{0}, \delta\right) \backslash B\left(x_{0}, t_{0} \delta\right)}\left(\frac{u_{0}}{\delta\left(1-t_{0}\right)}\right)^{2} \mathrm{~d} x, \\
& =\omega_{N} \delta^{N-2}\left(1-t_{0}^{N}\right) \frac{u_{0}^{2}}{\left(1-t_{0}\right)^{2}},
\end{aligned}
$$

where $\omega_{N}$ is the volume of unit sphere in $\mathbb{R}^{N}$, and $\delta, u_{0}$ are defined in $\left(f_{4}\right)$. Thus, $\left\|u_{t_{0}}\right\|$ is a positive constant which follows from (37). In the following, assume that $0<\mu<1$. Therefore, from (25) and the condition $\left(h_{1}\right)$,

$$
\left\|u_{t_{0}}\right\| H\left(\left\|u_{t_{0}}\right\|\right)-\widehat{H}\left(\left\|u_{t_{0}}\right\|\right)+\mu \int_{\Omega} \tau(w) u_{t_{0}} \mathrm{~d} x \leq \bar{A},
$$

where $\bar{A}$ is a positive constant.

By $\left(f_{2}\right),\left(f_{4}\right)$ and (32) and (36) we have

$$
\begin{aligned}
& \int_{\Omega} F\left(x, u_{t_{0}}\right) \mathrm{d} x, \\
& =\int_{U\left(x_{0}, \delta\right) \backslash U\left(x_{0}, t_{0} \delta\right)} F\left(x, \frac{u_{0}}{\delta\left(1-t_{0}\right)}\left(\delta-\left|x-x_{0}\right|\right)\right) \mathrm{d} x \\
& \quad+\int_{U\left(x_{0}, t_{0} \delta\right)} F\left(x, u_{t_{0}}\right) \mathrm{d} x \\
& \geq \int_{U\left(x_{0}, t_{0} \delta\right)} \eta_{0} \mathrm{~d} x-\int_{U\left(x_{0}, \delta\right) \backslash U\left(x_{0}, t_{0} \delta\right)}\left[\alpha\left|u_{0}\right|+\frac{\beta}{q}\left|u_{0}\right|^{q}\right] \mathrm{d} x \\
& \geq \omega_{N} \delta^{N} t_{0}^{N} \eta_{0}-\int_{U\left(x_{0}, \delta\right) \backslash U\left(x_{0}, t_{0} \delta\right)} M_{1} \mathrm{~d} x \\
& =\omega_{N} \delta^{N}\left(t_{0}^{N} \eta_{0}-M_{1}+M_{1} t_{0}^{N}\right),
\end{aligned}
$$

where $\eta_{0}$ is defined in $\left(f_{4}\right)$. Note that $0<\mu<1$, it follows from (16), (18), (38), and (39) that

$$
\begin{aligned}
I_{\lambda, \mu}\left(u_{t_{0}}\right)= & \left\|u_{t_{0}}\right\| H\left(\left\|u_{t_{0}}\right\|\right)-\hat{H}\left(\left\|u_{t_{0}}\right\|\right)-\lambda \int_{\Omega} F\left(x, u_{t_{0}}\right) \mathrm{d} x \\
& +\mu \int_{\Omega} \tau(w) u_{t_{0}} \mathrm{~d} x \leq \bar{A}-\lambda \omega_{N} \delta^{N}\left(\eta_{0} t_{0}^{N}-M_{1}+t_{0}^{N} M_{1}\right) .
\end{aligned}
$$

By (35), we obtain that

$$
\frac{M_{1}}{\eta_{0}+M_{1}}<t_{0}^{N}<1
$$

Thus,

$$
\eta_{0} t_{0}^{N}-M_{1}+t_{0}^{N} M_{1}=\left(t_{0}^{N}-\frac{M_{1}}{\eta_{0}+M_{1}}\right)\left(\eta_{0}+M_{1}\right)>0 .
$$

Therefore, there exists $\lambda_{\star}>0$, such that

$$
I_{\lambda, \mu}\left(u_{t_{0}}\right)\langle 0, \forall \lambda\rangle \lambda_{*} .
$$

By Proposition 2, the function $I_{\lambda, \mu}$ is bounded from below. By Proposition 3, the function $I_{\lambda, \mu}$ satisfies the (PS) condition. Thus, $I_{\lambda, \mu}$ attains its global minimum at some $u_{\lambda, \mu} \in H_{0}^{1}(\Omega)$ by Theorem 4. Obviously,

$$
I_{\lambda, \mu}\left(u_{\lambda, \mu}\right) \leq I_{\lambda, \mu}\left(u_{t_{0}}\right)\langle 0, \quad \forall \lambda\rangle \lambda_{*} .
$$

Next, we will prove the existence of the second critical point of $I_{\lambda, \mu}$ via the Mountain Pass theorem (see Theorem 2).

By the condition $\left(f_{3}\right)$ for any $\varepsilon>0$, there exists $t_{*}>0$, such that

$$
\frac{F(x, t)}{|t|^{\gamma}} \leq \chi(x)+\varepsilon, \quad \forall t \in\left[-t_{*}, t_{*}\right] .
$$

It follows from $\left(f_{2}\right)$ that for all $|t|>t_{*}$,

$$
|F(x, t)| \leq\left(\alpha(x)+\beta(x)|t|^{q-1}\right)|t| \leq\left(\frac{\alpha(x)}{t_{*}^{\gamma-1}}+\frac{\beta(x)}{t_{*}^{\gamma-q}}\right)|t|^{\gamma} .
$$

From (45) and (46), we obtain that for all $t \in \mathbb{R}$ and a.e. $x \in \Omega$,

$$
|F(x, t)| \leq\left(\chi(x)+\varepsilon+\frac{\alpha(x)}{t_{*}^{\gamma-1}}+\frac{\beta(x)}{t_{*}^{\gamma-q}}\right)|t|^{\gamma} .
$$

By the conditions $\left(h_{1}\right)$ and $\left(h_{2}\right)$, we obtain

$$
\lim _{t \rightarrow 0} \frac{t H(t)-\hat{H}(t)}{t^{2}}=\lim _{t \rightarrow 0} \frac{h(t)}{2}=\frac{h(0)}{2}>\frac{a}{2},
$$

and then there exists a positive constant $\bar{t}$ such that for all $t \in(0, \bar{t})$,

$$
t H(t)-\widehat{H}(t)>\frac{a}{2} t^{2} .
$$

Therefore, by (47) and (49), the Hölder inequality and the Sobolev embedding theorem, for all $\lambda>\lambda_{*}$ and $u \in \mathscr{K}_{\psi} \cap\left\{\|u\| \leq \bar{t}: u \in H_{0}^{1}(\Omega)\right\}$, we have

$$
I_{\lambda, \mu}(u)=\varphi_{\lambda, \mu}(u) \geq \frac{a}{2}\|u\|^{2}-\lambda c_{4}\|u\|^{\gamma}-\mu c_{5}\|u\|,
$$

where $c_{4}$ and $c_{5}$ are positive constants.

Denote

$$
\begin{aligned}
\Lambda & =\min \left\{\frac{1}{2}\left\|u_{t_{0}}\right\|,\left(\frac{a}{4 \lambda c_{4}}\right)^{1 / \gamma-2}, \bar{t}\right\}, \\
\mu_{*} & =\min \left\{\frac{a}{8 c_{5}} \Lambda, \frac{1}{2}\right\} .
\end{aligned}
$$

Let $\lambda>\lambda_{*}$ and $\mu<\mu_{*}$. Note that $\gamma>\max \{2, q\}$. It follows from (50) that 


$$
I_{\lambda, \mu}(u) \geq \frac{a}{8} \Lambda^{2}>0, \quad \forall u \in H_{0}^{1}(\Omega),\|u\|=\Lambda
$$

Obviously, $I_{\lambda, \mu}(0)=0$ and

$$
I_{\lambda, \mu}\left(u_{t_{0}}\right)<0,\left\|u_{t_{0}}\right\|>\Lambda
$$

By Proposition 3 and Theorem 2, there exists a critical point $v_{\lambda, \mu}$ of $I_{\lambda, \mu}$. We notice that the $v_{\lambda, \mu}$ cannot be trivial because

$$
I_{\lambda, \mu}\left(v_{\lambda, \mu}\right) \geq \frac{a}{8} \Lambda^{2}>0
$$

By Proposition 1, we conclude that $u_{\lambda, \mu}$ and $v_{\lambda, \mu}$ are two solutions of the Kirchhoff-type variational inequality (4). It follows from (44) and (54) that $I_{\lambda}\left(u_{\lambda}\right)<0<I_{\lambda}\left(v_{\lambda}\right)$. The proof is complete.

By Theorem 3, we have

Theorem 4. Let $\mathcal{U}$ be a nonempty bounded closed and convex subset of the space $L^{q}(\Omega)\left(1<q<\min \left\{4,2^{*}\right\}\right)$. If $f: \Omega \times \mathbb{R} \longrightarrow \mathbb{R}$ satisfies $\left(f_{1}\right)-\left(f_{4}\right)$ and $h(s)=a+b s^{2}$, $\tau: \mathcal{U} \longrightarrow L^{q^{\prime}}(\Omega)$ is a weakly continuous mapping such that $\tau(\mathcal{U})$ is a bounded set. Then, there exist $\lambda_{*}>0,0<\mu_{*}<1$, such that for each $\lambda>\lambda_{*}, \mu<\mu_{*}$, the Kirchhoff-type variational inequality (20) has two solutions $u_{\lambda}, v_{\lambda}$, satisfying $I_{\lambda}\left(u_{\lambda}\right)<0<I_{\lambda}\left(v_{\lambda}\right)$.

\section{Existence of an Optimal Control Governed by a Kirchhoff-Type Variational Inequality}

This section is concerned with the existence results of an optimal control for the optimal control problem $(\mathscr{P})$.

Lemma 1. Suppose $g(x, t): \Omega \times \mathbb{R} \longrightarrow \mathbb{R}$ satisfies

$\left(g_{1}\right) g(x, t)$ is measurable in $x$ for every $t \in \mathbb{R}$ and continuous in $t$ for a.e. $x \in \Omega$;

$\left(g_{2}\right)$ There exist positive constants $C_{i}, i=1,2$, such that

$$
|g(x, t)| \leq C_{1}+C_{2}|t|^{r}, \quad \forall(x, t) \in \Omega \times \mathbb{R} .
$$

where $1<r<2 *$.

Then, the functional $\mathbf{G}$ defined by

$$
\mathbf{G}(u)=\int_{\Omega} g(x, u) \mathrm{d} x, \quad u \in L^{r}(\Omega),
$$

is continuous.

Proof. Let $\left\{u_{n}\right\}$ be a sequence in $L^{r}(\Omega)$, such that $u_{n} \longrightarrow u_{0}$ as $n \longrightarrow+\infty$. Since $g$ satisfies the conditions $\left(g_{1}\right)$ and $\left(g_{2}\right)$, the operator $u \longmapsto g(x, u)$ from $L^{r}(\Omega)$ to $L^{1}(\Omega)$ is continuous. Therefore, we have

$$
\int_{\Omega} g\left(x, u_{n}\right) \mathrm{d} x \longrightarrow \int_{\Omega} g(x, u) \mathrm{d} x,
$$

as $n \longrightarrow \infty$. The proof is complete.

Lemma 2. Suppose $l(x, t): \Omega \times \mathbb{R} \longrightarrow \mathbb{R}$ is $C^{1}$ and for almost all $x \in \Omega, l(x, t)$ is convex with respect to $t \in \mathbb{R}$, and there exist positive constants $C_{i}, i=3, \ldots, 6$, such that

$$
\begin{gathered}
\left(l_{1}\right)|l(x, t)| \leq C_{3}+C_{4}|t|^{q}, \quad \forall(x, t) \in \Omega \times \mathbb{R}, \\
\left(l_{2}\right)\left|\frac{\partial l(x, t)}{\partial t}\right| \leq C_{5}+C_{6}|t|^{q-1}, \quad \forall(x, t) \in \Omega \times \mathbb{R},
\end{gathered}
$$

where $1<q<2 *$. Then, the functional $\mathbf{L}$ defined by

$$
\mathbf{L}(w)=\int_{\Omega} l(x, w) \mathrm{d} x, \quad w \in L^{q}(\Omega),
$$

is weakly lower semicontinuous.

Proof. Let $\left\{w_{n}\right\}$ be a sequence in $L^{q}(\Omega)$, such that $w_{n} \longrightarrow w_{0}$ as $n \longrightarrow+\infty$. Since $l(x, t)$ is $C^{1}$ and satisfies the conditions $\left(l_{1}\right)$ and $\left(l_{2}\right), \mathbf{L}$ is Gâteaux differentiable in the space $L^{q}(\Omega)$ and $\mathbf{L}^{\prime}\left(w_{0}\right) \in L^{q^{\prime}}(\Omega)$ (the dual space of $L^{q}(\Omega)$ ). Note that for each $x \in \Omega, l(x,$.$) is convex, and then$

$$
\mathbf{L}\left(w_{n}\right) \geq \mathbf{L}\left(w_{0}\right)+\left\langle\mathbf{L}^{\prime}\left(w_{0}\right), w_{n}-w_{0}\right\rangle .
$$

As $w_{n} \longrightarrow w_{0}$, from the inequality mentioned above by taking limits, we obtain

$$
\liminf _{n \longrightarrow+\infty} \mathbf{L}\left(w_{n}\right) \geq \mathbf{L}\left(w_{0}\right)
$$

The proof is complete.

Theorem 5. Let $\mathcal{U}$ be a nonempty bounded closed and convex subset of the space $L^{q}(\Omega)\left(1<q<\min \left\{4,2^{*}\right\}\right)$. Assume that $h: \mathbb{R} \longrightarrow \mathbb{R}$ satisfies the conditions $\left(h_{1}\right)-\left(h_{3}\right), f: \Omega \times$ $\mathbb{R} \longrightarrow \mathbb{R}$ satisfies the conditions $\left(f_{1}\right)-\left(f_{4}\right)$, $g: \Omega \times \mathbb{R} \longrightarrow \mathbb{R}$ satisfies $\left(g_{1}\right),\left(g_{2}\right), \quad \tau: \mathcal{U} \longrightarrow L^{q^{\prime}}(\Omega)$ a weakly continuous mapping such that $\tau(\mathcal{U})$ is a bounded set, and $g(x, t): \Omega \times \mathbb{R} \longrightarrow \mathbb{R}$ and $l: \Omega \times \mathbb{R} \longrightarrow \mathbb{R}$ satisfy all the conditions in Lemma 1 and Lemma 2, respectively. There exist $\lambda_{*}>0, \quad 0<\mu_{*}<1$, such that for each $\lambda>\lambda_{*}, \mu<\mu_{*}$, there exists an optimal control $\left(w_{0}, u_{0}\right) \in \mathcal{U} \times S\left(w_{0}\right)$ for the optimal control problem $(\mathscr{P})$.

Proof. From Theorem 3, there exist $\lambda_{*}>0,0<\mu_{*}<1$, such that for each $\lambda>\lambda_{*}, \mu<\mu_{*}, S(w) \neq \phi$, where $S(w)$ is the solution set of the variational inequality (4).

Let $\left\{\left(w_{n}, u_{n}\right)\right\}_{n \in N}\left(u_{n} \in S\left(w_{n}\right)\right)$ be a minimizing sequence for problem $(\mathscr{P})$ such that

$$
\lim _{n \longrightarrow \infty} \mathbf{J}\left(w_{n}, u_{n}\right)=\inf _{(w, u) \in \mathcal{U} \times S(w)} \mathbf{J}(w, u)
$$

where the cost function $\mathbf{J}$ is defined by (2).

As $u_{n} \in S\left(w_{n}\right)$, we have

$$
\begin{aligned}
& h\left(\left\|u_{n}\right\|\right) \int_{\Omega} \nabla u_{n}\left(\nabla v-\nabla u_{n}\right) \mathrm{d} x \\
& \quad \geq \lambda \int_{\Omega} f\left(x, u_{n}\right)\left(v-u_{n}\right) \mathrm{d} x-\mu \int_{\Omega} \tau\left(w_{n}\right)\left(v-u_{n}\right) \mathrm{d} x, \quad \forall v \in \mathscr{K}_{\psi} .
\end{aligned}
$$

We claim that $\left\{\left(w_{n}, u_{n}\right)\right\}_{n \in N}$ is bounded. In fact, as $U$ is bounded, $\left\{w_{n}\right\}$ is bounded. Let $v=0$ in (63). We get 


$$
\begin{aligned}
h\left(\left\|u_{n}\right\|\right)\left\|u_{n}\right\|^{2} \leq & \lambda \int_{\Omega} f\left(x, u_{n}\right) u_{n} \mathrm{~d} x-\mu \int_{\Omega} \tau\left(w_{n}\right) u_{n} \mathrm{~d} x \\
\leq & \lambda \int_{\Omega}\left|f\left(x, u_{n}\right) u_{n}\right| \mathrm{d} x+\mu \int_{\Omega} \tau\left(w_{n}\right) u_{n} \mathrm{~d} x \\
\leq & \lambda \int_{\Omega} \alpha\left|u_{n}\right|+\beta\left|u_{n}\right|^{q} \mathrm{~d} x+\mu \int_{\Omega} \tau\left(w_{n}\right) u_{n} \mathrm{~d} x \\
\leq & \lambda\left(\|\alpha\|_{L^{\infty}}\left\|u_{n}\right\|_{L^{1}}+\|\beta\|_{L^{\infty}}\left\|u_{n}\right\|_{L^{q}}^{q}\right) \\
& +\mu\left\|\tau\left(w_{n}\right)\right\|_{L^{q^{\prime}}}\left\|u_{n}\right\|_{L^{q}} \\
\leq & c_{6}(\lambda+\mu)\left\|u_{n}\right\|+c_{7}\left\|\lambda u_{n}\right\|^{q},
\end{aligned}
$$

where $c_{6}$ and $c_{7}$ are positive constants. As $1<q<4$ and $h$ satisfies $\left(h_{1}\right)-\left(h_{3}\right),\left\{u_{n}\right\}$ is bounded.

Since $L^{q}(\Omega)$ and $H_{0}^{1}(\Omega)$ are reflexive spaces, there exists a weakly convergent subsequence of $\left\{\left(w_{n}, u_{n}\right)\right\}$. Without loss of generality, we may assume $\left(w_{n}, u_{n}\right) \longrightarrow\left(w_{0}, u_{0}\right)$ as $n \longrightarrow \infty$. Hence, $w_{n} \longrightarrow w_{0}$ in $L^{q}(\Omega)$ and $u_{n} \longrightarrow u_{0}$ in $H_{0}^{1}(\Omega)$ as $n \longrightarrow \infty$. Since $\mathcal{U}$ and $\mathscr{K}_{\psi}$ are weakly closed sets, $w_{0} \in \mathscr{U}$ and $u_{0} \in \mathscr{K}_{\psi}$. Then, let $v=u_{0}$ in (63). We get

$$
\begin{aligned}
& h\left(\left\|u_{n}\right\|\right) \int_{\Omega} \nabla u_{n} \nabla\left(u_{0}-u_{n}\right) \mathrm{d} x \geq \lambda \int_{\Omega} f\left(x, u_{n}\right)\left(u_{0}-u_{n}\right) \mathrm{d} x \\
& \quad-\mu \int_{\Omega} \tau\left(w_{n}\right)\left(u_{0}-u_{n}\right) \mathrm{d} x .
\end{aligned}
$$

Since $f$ satisfies the conditions $\left(f_{1}\right)-\left(f_{4}\right)$ and $\tau$ is bounded,

$$
\begin{array}{r}
\lim _{n \rightarrow \infty} \lambda \int_{\Omega} f\left(x, u_{n}\right)\left(u_{0}-u_{n}\right) \mathrm{d} x=0, \\
\lim _{n \rightarrow \infty} \int_{\Omega} \tau\left(w_{n}\right)\left(u_{0}-u_{n}\right) \mathrm{d} x=0 .
\end{array}
$$

Then, from (65) and (66), we have

$$
\limsup _{n \rightarrow \infty} h\left\|\left(u_{n}\right)\right\| \int_{\Omega} \nabla u_{n} \nabla\left(u_{n}-u_{0}\right) \mathrm{d} x \leq 0 .
$$

Since $\left\{u_{n}\right\}$ is bounded and $h$ satisfies $\left(h_{1}\right)$ and $\left(h_{2}\right)$,

$$
\limsup _{n \rightarrow \infty} \int_{\Omega} \nabla u_{n} \nabla\left(u_{n}-u_{0}\right) \mathrm{d} x \leq 0 .
$$

Note that $u_{n} \longrightarrow u_{0}$ in $H_{0}^{1}(\Omega)$ as $n \longrightarrow \infty$, and we have

$$
\lim _{n \longrightarrow \infty} \int_{\Omega} \nabla u_{0} \nabla\left(u_{n}-u_{0}\right) \mathrm{d} x=0 .
$$

By (68) and (69), we get

$$
\limsup _{n \rightarrow \infty} \int_{\Omega}\left|\nabla\left(u_{n}-u_{0}\right)\right|^{2} \mathrm{~d} x \leq 0 .
$$

Thus, $u_{n} \longrightarrow u_{0}$ in $H_{0}^{1}(\Omega)$ as $n \longrightarrow+\infty$. Therefore, by (63), we have

$$
\begin{aligned}
& h\left(\left\|u_{0}\right\|\right) \int_{\Omega} \nabla u_{0}\left(\nabla v-\nabla u_{0}\right) \mathrm{d} x \geq \lambda \int_{\Omega} f\left(x, u_{0}\right)\left(v-u_{0}\right) \mathrm{d} x \\
& \quad-\int_{\Omega} \tau(w)\left(v-u_{0}\right) \mathrm{d} x, \quad \forall v \in \mathscr{K}_{\psi},
\end{aligned}
$$

that is, $u_{0} \in S\left(w_{0}\right)$. Denote

$$
\begin{array}{ll}
\mathbf{G}(u)=\int_{\Omega} g(x, u) \mathrm{d} x, & u \in \mathscr{K}_{\psi}, \\
\mathbf{L}(w)=\int_{\Omega} l(x, w) \mathrm{d} x, & w \in \mathcal{U} .
\end{array}
$$

Since $u_{n} \longrightarrow u_{0}$ in $H_{0}^{1}(\Omega)$ as $n \longrightarrow+\infty$ and the embedding $H_{0}^{1}(\Omega) \hookrightarrow L^{r}(\Omega)$ is continuous compact $\left(1<r<2^{*}\right), u_{n} \longrightarrow u_{0}$ in $L^{r}(\Omega)$ as $n \longrightarrow \infty$. By Lemma 1 and Lemma 2, G: $\mathscr{K}_{\psi} \longrightarrow \mathbb{R}$ is continuous and L: $L^{q}(\Omega) \longrightarrow \mathbb{R}$ is weakly lower semicontinuous. Therefore,

$$
\begin{aligned}
\mathbf{J}\left(w_{0}, u_{0}\right) & =\mathbf{G}\left(u_{0}\right)+\mathbf{L}\left(w_{0}\right) \\
& \leq \liminf _{n \longrightarrow+\infty}\left(\mathbf{G}\left(u_{n}\right)+\mathbf{L}\left(w_{n}\right)\right), \\
& =\liminf _{n \longrightarrow+\infty} \mathbf{J}\left(w_{n}, u_{n}\right), \\
& =\inf _{w \in \mathcal{U}, u \in S(w)} \mathbf{J}(w, u) .
\end{aligned}
$$

That is, $\left(w_{0}, u_{0}\right) \in \mathcal{U} \times S\left(w_{0}\right)$ is an optimal control for problem $(\mathscr{P})$. The proof is complete.

Theorem 6. Let $\mathcal{U}$ be a nonempty bounded closed and convex subset of the space $L^{q}(\Omega)\left(1<q<\min \left\{4,2^{*}\right\}\right)$. Assume that $h$ satisfies the conditions $\left(h_{1}\right)-\left(h_{3}\right), f$ satisfies the conditions $\left(f_{1}\right)-\left(f_{4}\right)$, and $\tau: \mathcal{U} \longrightarrow L^{q^{\prime}}(\Omega)$ is a weakly continuous mapping such that $\tau(\mathcal{U})$ is a bounded set. Let $1<r<p^{*}, g(u)=C_{7}\left|u-u_{d}\right|^{r}$, and $l(w)=C_{8}\left|w-w_{d}\right|^{q}$, for some constants $C_{7}$ and $C_{8}$, where $u_{d} \in L^{r}(\Omega), w_{d} \in L^{q}(\Omega)$. There exist $\lambda_{*}>0,0<\mu_{*}<1$, such that for each $\lambda>\lambda_{*}, \mu<\mu_{*}$, there exists an optimal control $\left(w_{0}, u_{0}\right) \in \mathcal{U} \times S\left(w_{0}\right)$ for the optimal control problem $(\mathscr{P})$.

Proof. Firstly, we claim that $J: \mathscr{U} \times \mathscr{K}_{\psi} \longrightarrow \mathbb{R}$ is weakly lower semicontinuous. In fact,

$$
\begin{aligned}
J(w, u) & =\int_{\Omega} g(u)+\int_{\Omega} l(w), \\
& =C_{7} \int_{\Omega}\left|u-u_{d}\right|^{r}+C_{8} \int_{\Omega}\left|w-w_{d}\right|^{q}, \\
& =C_{7}\left\|u-u_{d}\right\|_{L^{r}}^{r}+C_{8}\left\|w-w_{d}\right\|_{L^{q}}^{q} .
\end{aligned}
$$

By the weakly lower semicontinuity of the norm, $J: L^{q}(\Omega) \times L^{r}(\Omega) \longrightarrow \mathbb{R}$ is weakly lower semicontinuous.

Let $\left(w_{n}, u_{n}\right) \longrightarrow\left(w_{0}, u_{0}\right) \in \mathscr{U} \times \mathscr{K}_{\psi}$. Then, $w_{n} \longrightarrow w_{0}$ in $\mathcal{U}$ and $u_{n} \longrightarrow u_{0}$ in $\mathscr{K}_{\psi}$. Since $1<r<2^{*}$, it follows from the Sobolev imbedding theorem that the imbedding $H_{0}^{1}(\Omega) \longrightarrow L^{r}(\Omega)$ is a compact imbedding. Hence, $u_{n} \longrightarrow u_{0} \quad$ in $\quad L^{r}(\Omega)$ and $\left(w_{n}, u_{n}\right) \longrightarrow\left(w_{0}, u_{0}\right)$ in $L^{q}(\Omega) \times L^{r}(\Omega)$. Since $J: L^{q}(\Omega) \times L^{r}(\Omega) \longrightarrow \mathbb{R}$ is weakly lower semicontinuous, $J: \mathcal{U} \times \mathscr{K}_{\psi} \longrightarrow \mathbb{R}$ is weakly lower semicontinuous. Then, similar to the rest of the proof given in Theorem 5, we obtain our conclusion. 


\section{Data Availability}

No data were used to support this study.

\section{Conflicts of Interest}

The authors declare that they have no conflicts of interest.

\section{Acknowledgments}

The authors are grateful to the referees for their valuable comments for improving the paper. This research was supported by National Natural Science Foundation of China (11771319 and 11971339).

\section{References}

[1] A. Azzollini, "The Kirchhoff equation in $\mathbb{R}^{N}$ perturbed by a local nonlinearity," International Journal of Difference Equations, vol. 25, pp. 543-554, 2012.

[2] C. O. Alves and G. M. Figueiredo, "Nonlinear perturbations of a periodic Kirchhoff equation in $\mathbb{R}^{N}$," Nonlinear Analysis: Theory, Methods \& Applications, vol. 75, no. 5, pp. 2750-2759, 2012.

[3] Z. Zhang and K. Perera, "Sign changing solutions of Kirchhoff type problems via invariant sets of descent flow," Journal of Mathematical Analysis and Applications, vol. 317, no. 2, pp. 456-463, 2006.

[4] Y. Deng, S. Peng, and W. Shuai, "Existence and asymptotic behavior of nodal solutions for the Kirchhoff-type problems in," Journal of Functional Analysis, vol. 269, no. 11, pp. 3500-3527, 2015.

[5] Y. S. Huang, Z. Liu, and Y. Z. Wu, "On finding solutions of a Kirchhoff type problem," Proceedings of the American Mathematical Society, vol. 144, pp. 3019-3033, 2016.

[6] J. Zhang, "The Kirchhoff type Schrödinger problem with critical growth," Nonlinear Analysis: Real World Applications, vol. 28, pp. 153-170, 2016.

[7] J. Lee, J.-M. Kim, and Y.-H. Kim, "Existence and multiplicity of solutions for Kirchhoff-Schrodinger type equations involving $p(x)$ - laplacian on the entire space $\mathbb{R}^{N}$," Nonlinear Analysis: Real World Applications, vol. 45, pp. 620-649, 2019.

[8] J. L. Lions, Quelwues Méthodes de Résolution des Problémes Aux Limites Non-Linéaires, Dunod-Gauthier-Villars, Paris, France, 1969.

[9] J. L. Lions, Optimal Control of Systems Governed by Partial Differential Equations, Springer-Verlag, Berlin, Germany, 1971.

[10] J. L. Lions, Some Aspects of the Optimal Control of Distributed Parameter Systems CBMS-NSF Regional Conference Series in Applied Mathematics, Society for Industrial and Applied Mathematics, Philadelphia, PA, USA, 1972.

[11] V. Barbu, "Optimal control of variational inequalities," Research Notes in Mathematics, vol. 100, Pitman, Boston, MA, USA, 1984.

[12] H. Lou, "On the regularity of an obstacle control problem," Journal of Mathematical Analysis and Applications, vol. 258, no. 1, pp. 32-51, 2001.

[13] H. Lou, "An optimal control problem governed by quasilinear variational inequalities," SIAM Journal on Control and Optimization, vol. 41, no. 4, pp. 1229-1253, 2002.

[14] Q. Chen, "Optimal control for semilinear evolutionary variational bilateral problem," Journal of Mathematical Analysis and Applications, vol. 277, no. 1, pp. 303-323, 2003.
[15] M. Bergounioux and S. Lenhart, "Optimal control of bilateral obstacle problems," SIAM Journal on Control and Optimization, vol. 43, no. 1, pp. 240-255, 2004.

[16] Y. Ye and Q. Chen, "Optimal control of the obstacle in a quasilinear elliptic variational inequality," Journal of Mathematical Analysis and Applications, vol. 294, no. 1, pp. 258272, 2004.

[17] Y. Y. Zhou, X. Q. Yang, and K. L. Teo, "The existence results for optimal control problems governed by a variational inequality," Journal of Mathematical Analysis and Applications, vol. 321, no. 2, pp. 595-608, 2006.

[18] Y. Y. Zhou, X. Q. Yang, and K. L. Teo, "Optimal control problems governed by a variational inequality via nonlinear Lagrangian methods," Optimization, vol. 55, no. 1-2, pp. 187-203, 2006.

[19] A. A. Khan and M. Sama, "Optimal control of multivalued quasi variational inequalities," Nonlinear Analysis: Theory, Methods \& Applications, vol. 75, no. 3, pp. 1419-1428, 2012.

[20] Q. Chen, D. Chu, R. C. E. Tan, and Y. Ye, "Double obstacle control problem for a quasilinear elliptic variational inequality with source term," Nonlinear Analysis: Real World Applications, vol. 18, pp. 108-120, 2014.

[21] Q. Chen, D. Chu, and R. C. E. Tan, "Optimal control of obstacle for quasi-linear elliptic variational bilateral problems," SIAM Journal on Control and Optimization, vol. 44, no. 3, pp. 1067-1080, 2005.

[22] S. Migorski, A. A. Khan, and S. D. Zeng, "Inverse problems for nonlinear quasi-variational inequalities with an application to implicit obstacle problems of p-Laplacian type," Inverse Problems, vol. 35, 2019.

[23] A. A. Khan, S. Migorski, and M. Sama, "Inverse problems for multi-valued quasi variational inequalities and noncoercive variational inequalities with noisy data," Optimization, vol. 68, no. 10, pp. 1897-1931, 2019.

[24] A. Szulkin, "Minimax principles for lower semicontinuous functions and applications to nonlinear boundary value problems," Annales de l'Institut Henri Poincare (C) Non Linear Analysis, vol. 3, no. 2, pp. 77-109, 1986.

[25] F. H. Clarke, Optimization and Nonsmooth Analysis, John Wiley-Interscience, New York, NY, USA, 1983. 